\title{
Crystallization Caught in the Act with Terahertz Spectroscopy: Non-Classical Pathway for L-(+)-Tartaric Acid
}

Amin Soltani ${ }_{,}^{[a, d]}$ Denis Gebauer, ${ }^{[b]}$ Lennart Duschek ${ }_{,}^{[a]}$ Bernd M. Fischer, ${ }^{[a, c]}$ Helmut Cölfen, ${ }^{*[b]}$ and Martin Koch*[a]

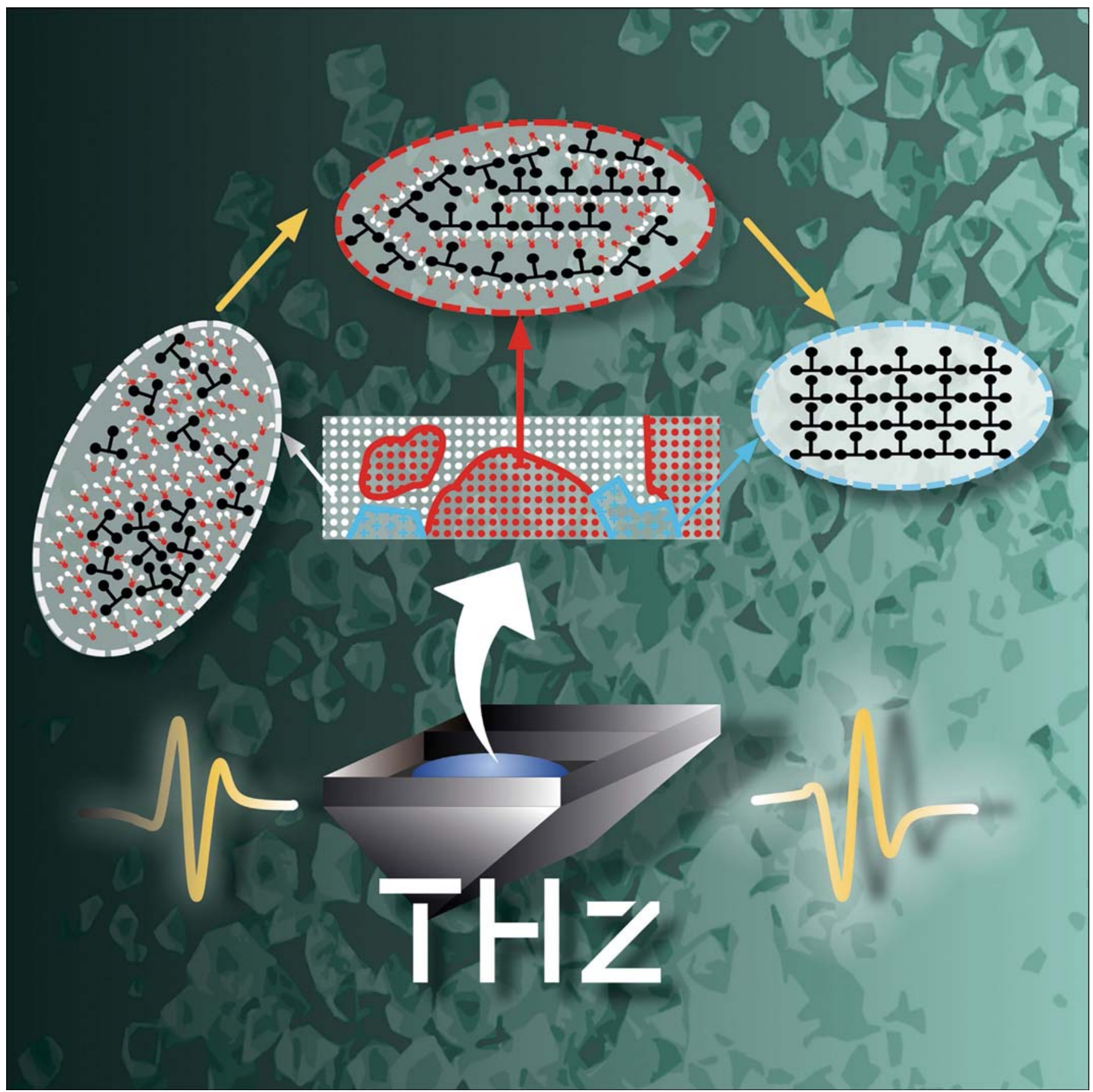


Abstract: Crystal formation is a highly debated problem. This report shows that the crystallization of L-(+)-tartaric acid from water follows a non-classical path involving intermediate hydrated states. Analytical ultracentrifugation indicates solution clusters of the initial stages aggregate to form an early intermediate. Terahertz spectroscopy performed during water evaporation highlights a transient increase in the absorption during nucleation; this indicates the recurrence of water molecules that are expelled from the intermediate phase. Besides, a transient resonance at $750 \mathrm{GHz}$, which can be assigned to a natural vibration of large hydrated aggregates, vanishes after the final crystal has formed. Furthermore, $\mathrm{THz}$ data reveal the vibration of nanosized clusters in the dilute solution indicated by analytical ultracentrifugation. Infrared spectroscopy and wideangle X-ray scattering highlight that the intermediate is not a crystalline hydrate. These results demonstrate that nanoscopic intermediate units assemble to form the first solvent-free crystalline nuclei upon dehydration.

Crystallization is a subject of intense research and discussion. ${ }^{[1-3]}$ On the one hand, classical nucleation theory considers the existence of a nucleus of critical size as the central transitional state for the direct formation of growing nuclei. The structure of this nucleus depends on the level of supersaturation. On the other hand, so-called non-classical approaches assume that the nucleation of crystals does not proceed directly, but includes intermediate states. ${ }^{[4-7]}$ In two-step nucleation, density fluctuations lead to the formation of a metastable dense liquid, in which crystals are nucleated at enhanced rates in a second step. ${ }^{[8]}$ According to the pre-nucleation cluster pathway, thermodynamically stable solute clusters are direct precursors to liquid-liquid separation, in which the nucleated nanodroplets aggregate and eventually yield amorphous intermediates by solidification as the precursors to crystals. ${ }^{[9]}$ Proposed intermediate states in the case of organic crystallization also include short-range supramolecular synthons, ${ }^{[10]}$ nucleation phases, and long range synthons. ${ }^{[2,6]}$ In this non-classical

[a] Dr. A. Soltani, L. Duschek, Dr. B. M. Fischer, Prof. M. Koch

Faculty of Physics and Material Sciences Center

Philipps-Universität Marburg

Renthof 5, 35032 Marburg (Germany)

E-mail:martin.koch@physik.uni-marburg.de

[b] Dr. D. Gebauer, Prof. H. Cölfen

Physical Chemistry

University of Konstanz

Universitätsstr. 10, 78457 Konstanz (Germany)

E-mail: Helmut.Coelfen@uni-konstanz.de

[c] Dr. B. M. Fischer

French-German Research Institute of Saint-Louis 68301 Saint-Louis (France)

[d] Dr. A. Soltani

Physikalisches Institut

Johann Wolfgang Goethe-Universitat

60438 Frankfurt am Main (Germany) view, the liquid-like clusters and intermediates in the pre- and post-nucleation stages, respectively, show distinct short-range orders but still contain solvent molecules. ${ }^{[1,4]}$ These solvent molecules are expelled once the system transforms into the final crystal, which is the energetically favorable state. Here, we primarily use far-infrared spectroscopy, which is sensitive to intermolecular and lattice vibrations. ${ }^{[11,12]}$ We employ terahertz time-domain spectroscopy in the attenuated total reflection (ATR) geometry. ${ }^{[13]}$ Terahertz spectroscopic investigations in combination with analytical ultracentrifugation (AUC), infrared (IR) and wide-angle X-ray scattering (WAXS) analyses allow us to delineate a comprehensive view on the multiple stages of the crystallization of L-(+)-tartaric acid from aqueous solution. Figure 1 illustrates this non-classical view and the different in-

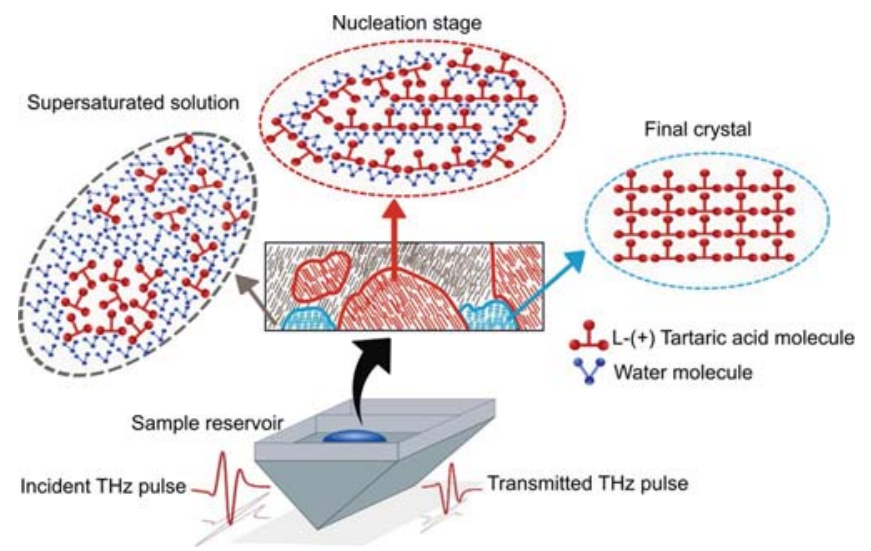

Figure 1. The balloons on the top show structural schemes of the molecular arrangement in the different stages. The center of the figure represents a small section of the probed volume at a fixed time. The probed volume contains a mixture of the different stages. As time proceeds, the relative contributions of these phases to the total volume changes. The sample reservoir with the evaporating solution positioned on top of a silicon prism is shown at the bottom of the figure. Also shown is the terahertz beam propagating through the prism.

termediate states in the context of our $\mathrm{THz}$ experiment. In the top left balloon, we see a schematic representation of the supersaturated solution in which individual molecules surrounded by hydration layers are crowded together. In addition, clusters form, which can contain water molecules. The middle balloon shows the nucleation stage (including pre- and post-nucleation stages), ${ }^{[9,14]}$ characterized by smaller or larger fragments of attached molecules, which could have the same arrangement and short-range order as in the final crystal. ${ }^{[2,15,16]}$ In between these fragments are still water molecules. The balloon on the right is a schematic representation of the final crystal after water expulsion. As time proceeds and water evaporates, different areas within the probed sample reservoir undergo the different stages at slightly different times, that is, not synchronously. Hence, at a given time, the probed volume can contain all different stages. This is shown in the middle of Figure 1 for a small section of the probed volume. The relative contribution of these stages to the total volume changes with time. The bottom of Figure 1 shows a scheme of the core of the setup as described in the methods section. 
The molecule or crystal studied here is anhydrous L-(+)-tartaric acid (L-(+)-TA), which in crystalline form exhibits a pronounced absorption resonance at $1.1 \mathrm{THz}$, which arises from an external rotation about the $a$ axis. ${ }^{[17,18]}$ L-(+)-TA powder was dissolved in distilled water to obtain a $1.060 \mathrm{M}$ solution. This solution was placed in the sample chamber on top of the ATR prism and was repeatedly measured over more than $60 \mathrm{~h}$ until the water was entirely evaporated and a crystal had formed. Figure $2 \mathrm{~A}$ shows a color-coded view of the $\mathrm{THz}$ absorption
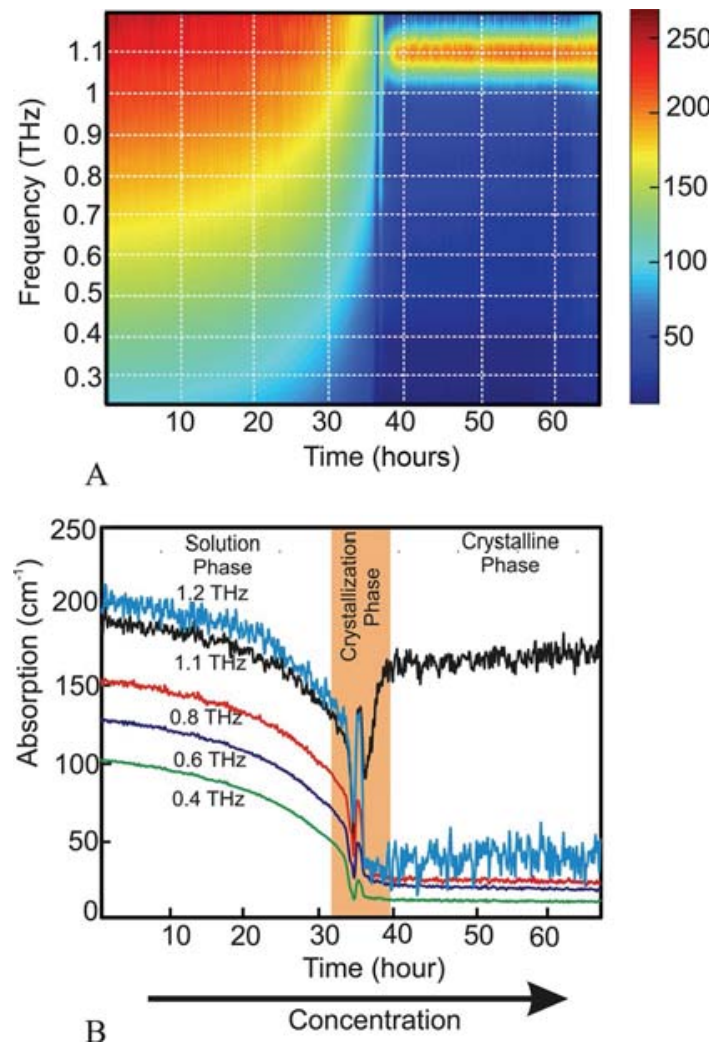

Figure 2. A) Color map of the $\mathrm{THz}$ absorption spectrum during crystallization and B) absorption coefficient of the solution versus time for selected frequencies. Between the solution phase and the crystalline phase we observe a transient stage that we call nucleation stage. As time progresses the concentration of the solution increases, but not necessarily in a linear relation with time.

spectrum in the time-frequency plane. This graph can be dissected into three stages. Initially, until approximately $32 \mathrm{~h}$, we are in the solution phase. During this stage, the absorption of bulk water dominates the spectrum. In the frequency window considered here, the absorption spectrum of bulk water is featureless and resembles a square-root shape. ${ }^{[19]}$ Figure S6 in the Supporting Information presents the absorption and refractive index of the water in the $\mathrm{THz}$ range, measured with our ATR THz-TDS setup. With increasing time, the concentration of bulk water decreases (due to evaporation) and, consequently, the ratio between bulk water and hydration shell water, which surrounds the freely floating molecules and clusters, drops. Hence, the time axis also reflects the concentration of the mixture. Since bulk water has a higher absorption than hydrated water in the lower $\mathrm{THz}$ range ${ }^{[20]}$ the total absorption of the solution decreases with time. At times after $39 \mathrm{~h}$, we are in the crystalline phase and the known absorption peak around 1.1 THz dominates the spectrum.

AUC analyses of the solution phase reveal the existence of a low concentration of clusters (0.5-3.3 wt \% increasing with experimental time), which are approximately $2.2 \mathrm{~nm}$ (see the Supporting Information, Table S1) in hydrodynamic diameter containing $>30$ TA molecules already in the initial homogeneous solution besides a majority of hydrated TA (see the Supporting Information). As time proceeds, these clusters and larger aggregates of $2.7 \mathrm{~nm}$ diameter can be observed, which likely represent the direct precursors to the intermediate formed in the nucleation stage, since their concentration increases with experimental time prior to nucleation. In addition, $\mathrm{THz}$ spectra obtained during the solution stage exhibit weak resonances, which could arise from clusters and aggregates evidenced by AUC (Figure $S 6$ in the Supporting Information). The nucleation stage lies between 32 and 39 h, where the $\mathrm{THz}$ data show two distinct interesting features. First, the absorption decreases abruptly, then shows a transient increase around $36 \mathrm{~h}$, which lasts only for one hour. The abrupt decrease and the transient increase are observable over a wide frequency range. To further illustrate this, we show in Figure $2 \mathrm{~B}$ the $\mathrm{THz}$ absorption versus time for different frequencies. Only at the position of the peak at $1.1 \mathrm{THz}$, the absorption in the final state is higher than that of the transient temporal peak around $36 \mathrm{~h}$.

The second interesting feature is an absorption peak around $750 \mathrm{GHz}$, which is present only during the nucleation stage. As this peak is not evident in the color-coded plot in Figure $2 \mathrm{~A}$, we plot a set of spectra for different times in Figure 3. The three red spectra in the background are obtained at different times in the solution phase. The three black spectra in the front clearly show the peak at $1.1 \mathrm{THz}$ and were recorded after the final crystal had formed. The three blue curves are obtained during the nucleation stage. The second blue curve shows a broadband transient increase, which we attribute to the reoccurrence of bulk water, as water molecules are released when a certain fraction of the probed volume transforms into the final crystal. ${ }^{[2]}$

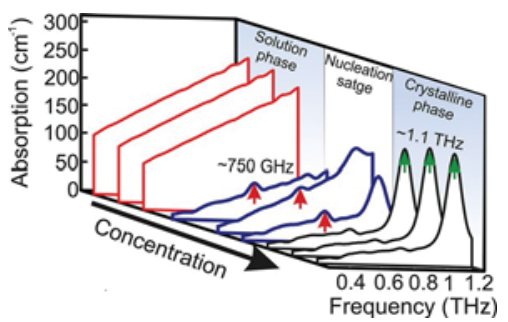

Figure 3. Set of absorption spectra for selected times. From left to right, the curves were recorded at (HH/MM) 7:00, 16:00, 26:00, 36:00, 36:30, 37:15, 38:00, 42:00, and 47:00. We recorded a spectrum every $3 \mathrm{~min}$. The curves shown represent an average of 11 subsequent spectra centered around the respective time; except for the 5 th spectrum shown (36:30), which represents an average of only 7 spectra due to the quick dynamics observed around this time. 
Furthermore, one observes the crystal resonance at $1.1 \mathrm{THz}$, the relative strength of which increases with time as the final crystals gain volume (cf. Figure 1). In addition, the data show the above-mentioned peak at $750 \mathrm{GHz}$, which we attribute to a natural vibration of entities of several inter-connected molecules surrounded by water molecules, as present in the nucleation stage. Indeed, additional IR spectroscopic and WAXS analyses of the nucleation stage as well as a THz ATR measurement on an aqueous solution of meso-tartaric acid monohydrate show that this feature cannot be due to the formation of an intermediate crystalline hydrate (see Figures S2, S3, and S7 in the Supporting Information). As time progresses and the final crystals constitute a continuously increasing fraction of the probed volume, the relative contribution of the $750 \mathrm{GHz}$ becomes continuously smaller. One notes that the probed volume does not homogeneously undergo the different phases of crystallization (cf. Figure 1). For example, the second blue curve in Figure 3 shows the $750 \mathrm{GHz}$ peak associated with the nucleation stage, the broadband absorption increase associated with water expulsion, and also a weak $1.1 \mathrm{THz}$ crystal resonance.

Two further experiments verify that the peak at $750 \mathrm{GHz}$ is associated with the presence of water molecules around smaller or larger fragments of assembled molecules. In these two experiments, we used pure methanol as a solvent for L-(+)-TA $(0.939 \mathrm{M})$. Methanol has a lower absorption coefficient than water in the $\mathrm{THz}$ range. ${ }^{[21]}$ Besides, methanol evaporates faster than water. Consequently, the crystallization process moves faster with methanol as solvent. In one case, the reservoir above the sample cell was purged with dry nitrogen. The corresponding spectra are shown in Figure $4 \mathrm{~A}$. In the solution phase, we again observe a rather featureless spectrum dominated by the absorption of methanol. In the crystalline phase, we now observe two natural vibrational resonances located at 1 and $1.16 \mathrm{THz}$. Evidently, the resulting crystal has a different structure and, hence, a different vibration spectrum than that obtained with water as a solvent. ${ }^{[22]}$ This is not surprising, as it is well known that the solvent used can influence the crystallization outcome in terms of the final crystal structure, that is, polymorphism. However, in this case, in which we ensure that almost no water molecules will enter the solution, we do not observe anything similar to the nucleation stage described above and also no transient peak. Figure 4C (black curve) exemplarily shows the absorption at $800 \mathrm{GHz}$, which looks clearly different to what was observed for the aqueous solution.

In the second experiment, the methanol/L-(+)-TA solution was exposed to ambient air. In this case, one again observes the transient peak at $750 \mathrm{GHz}$. It is well known that methanol is highly hygroscopic. ${ }^{[23,24]}$ Hence, after some time, the methanol/L-(+)-TA solution will also contain a significant amount of water molecules that can form a hydration shell around the L(+)-TA molecules and can be in between smaller or larger fragments of attached molecules during the nucleation stage. In the end, we again observe the crystal peak at $1.1 \mathrm{THz}$, suggesting that we obtain the same crystal structure as with the water solution. Besides, the temporal behavior of the absorption at $800 \mathrm{GHz}$ looks similar again to the case in which pure water
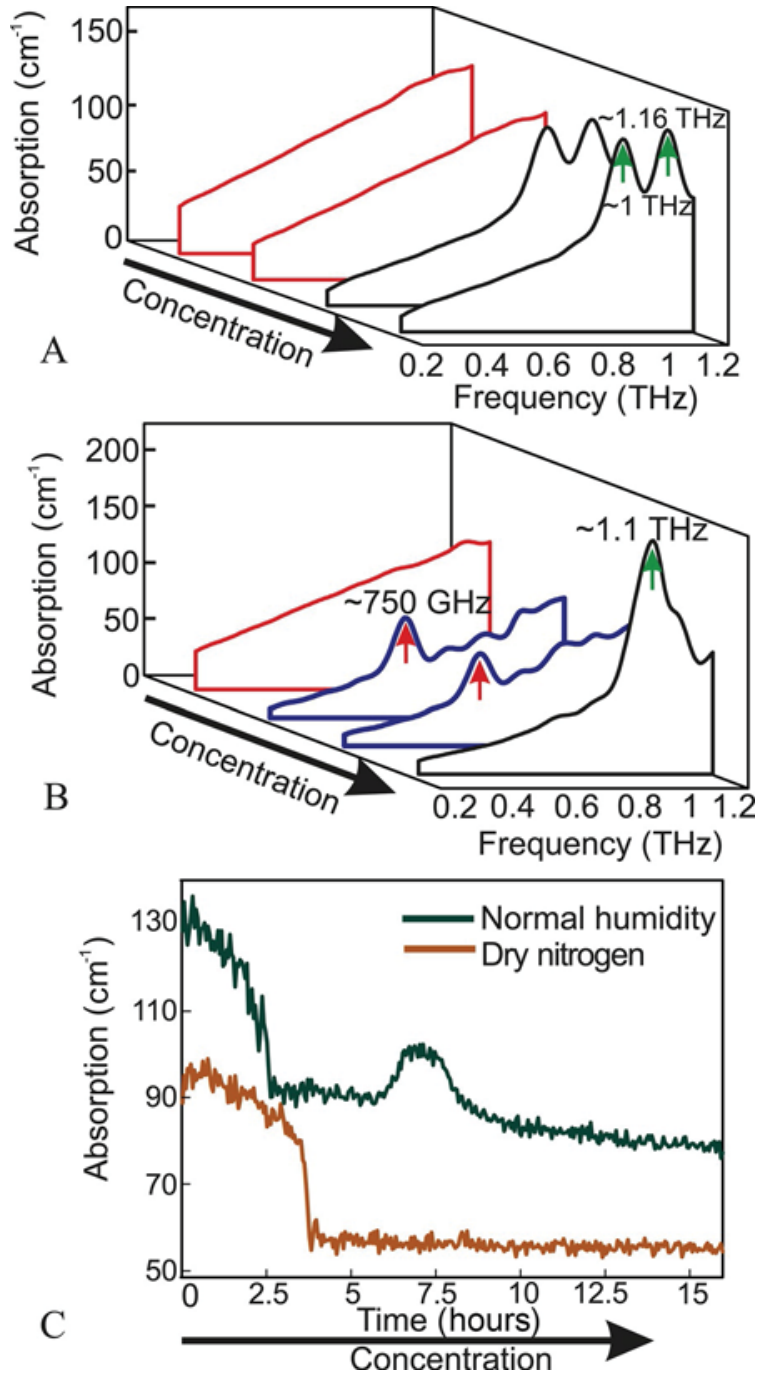

Figure 4. $\mathrm{THz}$ absorption of the L-(+)-tartaric acid solved in methanol. A) $\mathrm{THz}$ absorption of the solution in dry nitrogen and B) $\mathrm{THz}$ absorption of the solution under normal humidity. In $(A)$, curves were recorded at $(\mathrm{HH}$ / MM) 1:00, 3:00, 6:30, 10:00 and in (B), at (HH/MM) 1:00, 3:15, 4:30, 10:00. Every spectrum was recorded within $3 \mathrm{~min}$. The curves show an average of 11 subsequent spectra centered around the respective time. C) shows the absorption comparison at $800 \mathrm{GHz}$ for both measurements.

served as solvent (Figure 4C, red curve). In particular, one observes again a transient increase directly before the final crystal phase is reached (Figure 4B,C), due to the expulsion of water during the transformation into the final crystal.

The experiments presented here demonstrate that the crystallization of L-(+)-tartaric acid in water follows a non-classical pathway via clusters that are already present in the starting solution. A second larger cluster is observed at later sampling stages, suggesting aggregation of the primary clusters, as was observed for $\mathrm{CaCO}_{3} \cdot{ }^{[25]}$ We observe a nucleation stage characterized by a transient absorption peak at $750 \mathrm{GHz}$, which we attribute to a natural vibration of aggregates of attached L(+)-TA molecules surrounded by water molecules. Upon transformation into the final crystal water is expelled. This is reflected in a transient broadband absorption increase. 
In conclusion, the crystallization of $\mathrm{L}-(+)$ tartaric acid in aqueous solution involves solution clusters and intermediate stages, and water plays a key role. Thus, $\mathrm{THz}$ absorption spectroscopy in an ATR geometry is a new technique that opens the door to a systematic exploration of crystallization pathways in different systems. This could also have great impact on the field of crystal engineering, because fundamental questions as to the mechanisms of crystallization can be addressed.

\section{Acknowledgements}

We thank Prof. Desiraju for careful discussion about the $\mathrm{THz}$ results and commenting the manuscript. We thank Rose Rosenberg, Johanna Scheck, and Masoud Farhadi-Khouzani for the AUC measurements and evaluation, and experimental assistance with WAXS and IR measurements, respectively. A.S. acknowledges funding from the German Academic Exchange Service (DAAD). D.G. thanks the Zukunftkolleg of the University of Konstanz for financial support.

\section{Conflict of interest}

The authors declare no conflict of interest.

Keywords: cluster compounds · crystallization process · L$(+)$-tartaric acid $\cdot$ non-classical nucleation $\cdot$ THz spectroscopy

[1] R. J. Davey, S. L. M. Schroeder, J. H. Ter Horst, Angew. Chem. Int. Ed. 2013, 52, 2166-2179; Angew. Chem. 2013, 125, 2220-2234.

[2] G. R. Desiraju, J. Am. Chem. Soc. 2013, 135, 9952-9967.

[3] J. J. De Yoreo, P. U. P. A. Gilbert, N. A. J. M. Sommerdijk, R. L. Penn, S. Whitelam, D. Joester, H. Zhang, J. D. Rimer, A. Navrotsky, J. F. Banfield, Science 2015, 349, aaa6760.
[4] D. Erdemir, A. Y. Lee, A. S. Myerson, Acc. Chem. Res. 2009, 42, 621-629.

[5] S. Parveen, R. J. Davey, G. Dent, R. G. Pritchard, Chem. Commun. 2005, 1531.

[6] G. R. Desiraju, Angew. Chem. Int. Ed. 2007, 46, 8342-8356; Angew. Chem. 2007, 119, 8492-8508.

[7] G. R. Desiraju, J. J. Vittal, A. Ramanan, Crystal Engineering: A Textbook, World Scientific, 2011.

[8] P. G. Vekilov, Prog. Cryst. Growth Charact. Mater. 2016, 62, 136-154.

[9] D. Gebauer, M. Kellermeier, J. D. Gale, L. Bergström, H. Cölfen, Chem. Soc. Rev. 2014, 43, 2348.

[10] G. R. Desiraju, Angew. Chem. Int. Ed. Engl. 1995, 34, 2311-2327; Angew. Chem. 1995, 107, $2541-2558$.

[11] M. Tonouchi, Nat. Photonics 2007, 1, 97-105.

[12] P. U. Jepsen, D. G. Cooke, M. Koch, Laser Photonics Rev. 2011, 5, 124 166.

[13] H. Hirori, K. Yamashita, M. Nagai, K. Tanaka, Jpn. J. Appl. Phys. 2004, 43, L1287-L1289.

[14] F. Schüth, P. Bussian, P. Ågren, S. Schunk, M. Lindén, Solid State Sci. 2001, 3, $801-808$.

[15] R. Banerjee, P. M. Bhatt, N. V. Ravindra, G. R. Desiraju, Cryst. Growth Des. 2005, 5, 2299-2309.

[16] J. J. De Yoreo, P. G. Vekilov, Rev. Min. Geochem 2003, 54, 57-93.

[17] B. Fischer, M. Hoffmann, H. Helm, G. Modjesch, P. U. Jepsen, Semicond. Sci. Technol. 2005, 20, S246-S253.

[18] E. M. Witko, T. M. Korter, J. Phys. Chem. A 2011, 115, 10052-10058.

[19] L. Thrane, R. H. Jacobsen, P. U. Jepsen, S. R. Keiding, Chem. Phys. Lett. 1995, 240, 330-333.

[20] T. Arikawa, M. Nagai, K. Tanaka, Chem. Phys. Lett. 2008, 457, $12-17$.

[21] Y. Yomogida, Y. Sato, R. Nozaki, T. Mishina, J. Mol. Liq. 2010, 154, 31 - 35.

[22] M. D. King, T. M. Korter, J. Phys. Chem. A 2010, 114, 7127-7138.

[23] A. Lee, C. K. Law, Combust. Sci. Technol. 1992, 86, 253-265.

[24] S. G. Lim, T. A. Nickerson, J. Dairy Sci. 1973, 56, 843-848.

[25] D. Gebauer, A. Volkel, H. Cölfen, Science 2008, 322, 1819-1822. 